\title{
Hernia inguinal encarcelada por impacto fecal de sigmoides en un lactante
}

\author{
Dr. Miguel Ángel Medina-Andrade, ${ }^{*}$ Dr. Antonio Francisco Gallardo-Meza, ${ }^{* *}$ Dr. Miguel Ángel Piña-Garay, ${ }^{*}$ \\ Dr. José Manuel González-Sánchez*
}

\section{RESUMEN}

Niño de dos meses de edad con hernia inguinal encarcelada de 20 horas de evolución sin historia de estreñimiento, acompañada de vómito. Se intentó la reducción manual de la hernia sin éxito. Se decidió operar. Durante la cirugía se halló el sigmoides sin datos de isquemia; contenía una masa fecal compacta causante de la encarcelación. El problema se resolvió con taxis caudocefálica, enemas y herniorrafia. El paciente tuvo buena evolución postoperatoria. Este caso es una rara forma de encarcelación de una hernia. No encontramos otro caso similar en la literatura.

Palabras clave: hernia inguinal encarcelada, estreñimiento, impactación fecal.

\section{ABSTRACT}

We present the case of a two month old male with incarcerated inguinal hernia, twenty hours of evolution with associated vomiting. There was no history of constipation. Manual reduction of the hernia was unsuccessful. During surgery the sigmoid was not ischemic; it contained a compact fecal mass which caused the incarceration. This condition was successfully resolved with caudoceophalic taxis, rectal enemas and hernioplasty. This patient had an unusual form of incarceration. We could not find a similar case in literature.

Key words: Incarcerated inguinal hernia, constipation, fecal impaction.

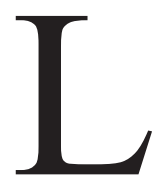

a hernia inguinal en menores de dos años tiene un alto riesgo de complicaciones que llega a ser hasta $11.8 \%$. La encarcelación es la más frecuente con $23.8 \%{ }^{1}$. El mayor riesgo ocurre en los primeros dos meses de vida. En estos pacientes es indispensable realizar una hernioplastia abierta con ligadura alta que tiene menor riesgo de complicaciones ${ }^{2}$. Sólo se recomienda la reducción por taxis en algunos pacientes bajo sedación ${ }^{3-5}$. Algunos casos como la hernia de Littre

* Médico adscrito del Servicio de Cirugía Pediátrica,

** Jefe del Servicio de Cirugía Pediátrica

Hospital General de Occidente

Servicio de Cirugía Pediátrica.

Correspondencia: Dr. Miguel Ángel Medina-Andrade. Av. Zoquipan \#1050. Colonia: Seattle Zapopan, Jalisco, México. C.P. 45170. Tel y fax: (33)3030 6354. E-mail: miguelcxp@yahoo.com.mx, miguelmedinaccv@gmail.com

Recibido: agosto, 2009. Aceptado: noviembre, 2009.

Este artículo debe citarse: Medina-Andrade MA, Gallardo-Meza AF, Piña-Garay MA y col. Hernia inguinal encarcelada por impacto fecal de sigmoides en un lactante. Acta Pediatr Mex 2010;31(1):29-31. La versión completa de este artículo también está disponible en: www.nietoeditores.com.mx
6, o la descrita por Amiand en 1735 pueden ocurrir a edades tempranas y deben considerarse en el diagnóstico diferencial ${ }^{7,8}$.

\section{INFORME DEL CASO}

Niño de dos meses de edad con una tumoración inguinal izquierda de 20 horas de evolución y vómitos de contenido alimentario con residuos de fórmula láctea no digerida. La abuela refiere que la madre del paciente padece alteraciones mentales y para alimentar al bebé preparaba una fórmula con el doble de la concentración normal. Se piensa que esto causó acumulo de materia fecal sólida en el sigmoides; no había historia previa de estreñimiento. Exploración física. El paciente tenía una tumoración de aproximadamente $2.5 \mathrm{~cm}$ de diámetro; sin cambio de coloración, ni eritema; indurada, dolorosa a la palpación, inmóvil. La transiluminación fue negativa; no fue posible reducirla manualmente. No había datos de irritación peritoneal; testículos descendidos. No se detectó alteración anal durante la inspección y canalización de gases al tacto rectal. Previo consentimiento informado se 
decidió operar para resolver el problema urgente de una hernia inguinal izquierda encarcelada. En la operación se halló edema tisular acentuado; el sigmoides se encontraba dentro del saco herniario; no mostraba isquemia; estaba ocupado por una masa fecal dura que impedía su retorno a la cavidad abdominal, (Figura 1). Se amplió el anillo inguinal interno y se realizó taxis caudocefálica de la masa fecal con reintroducción del asa intestinal a la cavidad abdominal. Se efectuó hernioplastia con la técnica de Ferguson modificada. La evolución postoperatoria fue satisfactoria. Se mantuvo al paciente en ayuno por dos horas; se hicieron enemas con fosfato de sodio $10 \mathrm{~mL} /$ $\mathrm{kg}$, con lo que se produjeron evacuaciones pétreas sin sangrado. Se inició la alimentación del niño con fórmula a dilución normal. Egresó por mejoría 24 horas después de la cirugía. A los ocho días, los familiares informaron que el niño tenía evacuaciones blandas dos veces diarias desde que salió del hospital.

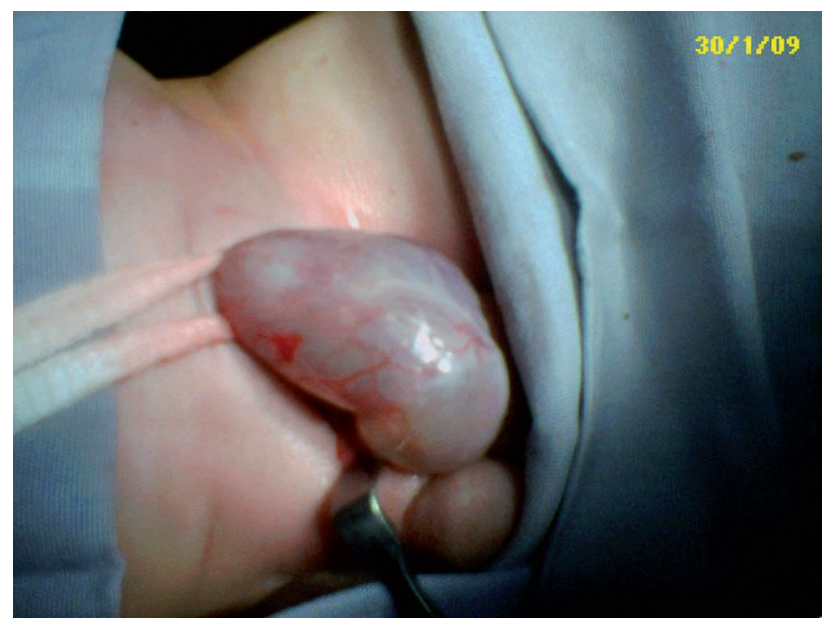

Figura 1. Saco herniario inguinal izquierdo.

\section{DISCUSIÓN Y ANÁLISIS}

Este es un caso raro de encarcelación del sigmoides por impacto fecal, que se diagnóstico al momento de la cirugía. La hernia se redujo manualmente por taxis una vez abierto el anillo inguinal profundo. La literatura señala que el mayor riesgo de encarcelación ocurre en los primeros dos meses de vida como el presente caso ${ }^{9}$. Su resolución quirúrgica fue satisfactoria. Las evacuaciones, se normalizaron cuando se corrigió la concentración de la fórmula láctea.

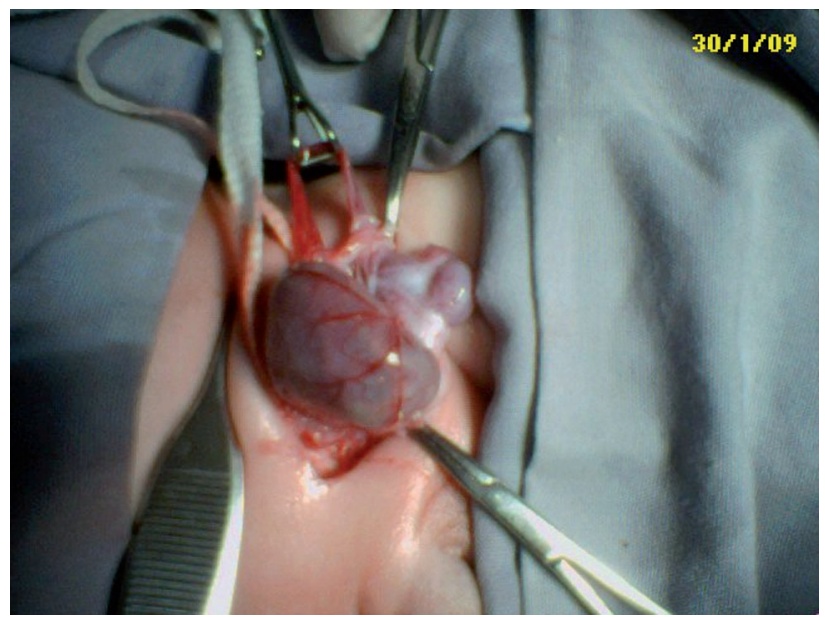

Figura 2. Masa fecal compacta que provocó la estrangulación del sigmoides.

Algunos autores sugieren alimentos ricos en sorbitol ${ }^{10}$ para el tratamiento inicial de la constipación de lactantes. Otros sugieren enemas, laxantes, polietilenglicol en caso de constipación refractaria, lo que tiene un costo elevado ${ }^{11,12}$. En nuestro paciente esto no fue necesario ya que al identificar y corregir la dilución de la fórmula las evacuaciones se normalizaron.

\section{CONCLUSIÓN}

Este caso es como una rara forma de hernia inguinal encarcelada. No encontramos otro caso parecido en la literatura de un bolo fecal compactado en sigmoides como causa de estrangulación en una hernia inguinal.

\section{BIBLIOGRAFÍA}

1. Zamakhshari M, To T, Guan J, Langer J. Risk of incarceration of inguinal hernia among infants and young children awaiting elective surgery. CMAJ. 2008;179(10):1001-5.

2. Brandt ML. Pediatric hernias. Surg Clin N Am. 2008;88:2743.

3. Glick PL, Boulanger SC. Inguinal hernias and hydroceles. In: Grossfeld JL, O`Neill JA, Coran AG, Fonkalsrud EW. Pediatric Surgery. Sixth ed. Philadelphia: Mosby; 2006. p. 1184-92.

4. Lloyd D. Inguinal and femoral hernia. In: Ziegler M, Azizkhan $\mathrm{R}$, Weber T. Operative pediatric surgery. New York: McGrawHill; 2003. p. 543-54.

5. McCollough M, Sharieff GQ. Abdominal pain in children. Pediatr Clin North Am. 2006; 53:107-37.

6. Akin M, Kurukahvecioglu O, Tezcaner T, Anadol AZ. Acute hemiscrotum due to inguinoscrotal Littre hernia: a case report. Am J Emerg Med. 2008;26:973.e3-973.e4.

7. Dilley A, Adams S. Acute scrotum caused by appendicitis. J 
Pediatr Surg. 1999;34:1183.

8. Doyle GS, McCowan C. Amyand hernia: a case of an unusual inguinal herniace. Am J Emerg Med. 2008;26:637.e5-6.

9. Louie JP. Essential diagnosis of abdominal emergencies in the first year of life. Emerg Med Clin North Am. 2007;25:1009-40.

10. Loening BV. Prevalence, symptoms and outcome of constipation in infants and toddlers. J Pediatr. 2005;146:359-63.
11. Biggs WS, Dery WH. Evaluation and treatment of constipation in infants and children. Am Fam Physician. 2006;73:469-77.

12. Liem O, Harman J, Benninga M, Kelleher K, Mousa H, Di Lorenzo C. Health utilization and cost impact of childhood constipation in the United States. J Pediatr. 2009;154:25862. 